\title{
Clusterin enhances AKT2-mediated motility of normal and cancer prostate cells through a PTEN and PHLPP1 circuit
}

\author{
Jessika Bertacchini ${ }^{1}$ ( ) $\mid$ Laura Mediani $^{1}$ | Francesca Beretti ${ }^{2}$ | Marianna Guida ${ }^{1}$ | \\ Aram Ghalali $^{3}$ | Federica Brugnoli ${ }^{4}$ | Valeria Bertagnolo ${ }^{4}$ | Emanuel Petricoin ${ }^{5}$ | \\ Francesco Poti $^{6}$ | Jessica Arioli ${ }^{1}$ | Laura Anselmi ${ }^{1}$ | Alessia Bari ${ }^{7}$ | \\ James McCubrey ${ }^{8}$ | Alberto M. Martelli ${ }^{9}$ | Lucio Cocco9 ${ }^{9}$ | Silvano Capitani ${ }^{4}$ | \\ Sandra Marmiroli ${ }^{1}$ (i)
}

${ }^{1}$ Department of Biomedical, Metabolic, and Neural Sciences, Section of Morphology, Signal Transduction Unit, University of Modena and Reggio
Emilia, Modena, Italy
${ }^{2}$ Department of Medicine, Surgery, Dentistry, and Morphology, University of Modena and Reggio Emilia, Modena, Italy
${ }^{3}$ Institute of Environment Medicine, Karolinska Institutet, Stockholm, Sweden
${ }^{4}$ Department of Morphology, Surgery, and Experimental Medicine, Section of Anatomy and Histology and LTTA Center, University of Ferrara,
Ferrara, Italy
${ }^{5}$ Center for Applied Proteomics \& Molecular Medicine, GMU, Fairfax, Virginia
${ }^{6}$ Department of Medicine and Surgery-Unit of Neurosciences, University of Parma, Parma, Italy
${ }^{7}$ Department of Diagnostic, Clinical Medicine and Public Health, Program of Innovative Therapy in Oncology and Hematology, University of
Modena and Reggio Emilia, Modena, Italy
${ }^{8}$ Department of Microbiology and Immunology, Brody School of Medicine at East Carolina University, Greenville, North Carolina
${ }^{9}$ Department of Biomedical and NeuroMotor Sciences, University of Bologna, Bologna, Italy

\section{Correspondence}

Jessika Bertacchini, Department of Biomedical, Metabolic and Neural Sciences, Section of Morphology, Signal Transduction Unit, University of Modena and Reggio Emilia, Modena 41124, Italy.

Email: jessika.bertacchini@unimore.it Sandra Marmiroli, Department of Biomedical, Metabolic and Neural Sciences, Section of Morphology, Signal Transduction Unit, University of Modena and Reggio Emilia, 41124 Modena, Italy. Email: sandra.marmiroli@unimore.it

\begin{abstract}
Clusterin (CLU) is a chaperone-like protein with multiple functions. sCLU is frequently upregulated in prostate tumor cells after chemo- or radiotherapy and after surgical or pharmacological castration. Moreover, CLU has been documented to modulate the cellular homolog of murine thymoma virus akt8 oncogene (AKT) activity. Here, we investigated how CLU overexpression influences phosphatidylinositol 3'-kinase (PI3K)/ AKT signaling in human normal and cancer epithelial prostate cells. Human prostate cells stably transfected with CLU were broadly profiled by reverse phase protein array (RPPA), with particular emphasis on the PI3K/AKT pathway. The effect of CLU overexpression on normal and cancer cell motility was also tested. Our results clearly indicate that CLU overexpression enhances phosphorylation of AKT restricted to isoform 2. Mechanistically, this can be explained by the finding that the phosphatase $\mathrm{PH}$ domain leucine-rich repeatcontaining protein phosphatase 1 (PHLPP1), known to dephosphorylate AKT2 at S474, is markedly downregulated by CLU, whereas miR-190, a negative regulator of PHLPP1, is upregulated. Moreover, we found that phosphatase and tensin homolog (PTEN) was heavily phosphorylated at the inhibitory site S380, contributing to the hyperactivation of AKT signaling. By keeping AKT2 phosphorylation high, CLU dramatically enhances the migratory behavior of prostate epithelial cell lines with different migratory and invasive phenotypes, namely prostate normal epithelial $1 \mathrm{~A}$ (PNT1A) and prostatic carcinoma 3 (PC3) cells. Altogether, our results unravel for the first time a circuit by which CLU can
\end{abstract}


switch a low migration phenotype toward a high migration phenotype, through miR-190dependent downmodulation of PHLPP1 expression and, in turn, stabilization of AKT2 phosphorylation.

KEYWORDS

AKT2, clusterin (CLU), miR-190, PHLPP1, prostate cancer

\section{1 | INTRODUCTION}

The many functions of the ubiquitously expressed protein clusterin (CLU) are mirrored by the multiplicity of its names (Apolipoprotein $\mathrm{J}$, testosterone-repressed prostate message 2 , and sulfate glyprotein-2 among others). Under physiological conditions, the predominant translation product of the human gene is a $80 \mathrm{kDa}$ heterodimeric, disulfide-linked, and highly glycosylated secretory protein, sCLU, mainly characterized by the chaperoning function as well as the cytoprotective activity (Bonacini et al., 2015; Choi-Miura, Takahashi, Nakano, Tobe, \& Tomita, 1992; Kim \& Choi, 2011; Leskov, Klokov, Li, Kinsella, \& Boothman, 2003; Prochnow et al., 2013; Pucci, Mazzarelli, Nucci, Ricci, \& Spagnoli, 2009; Rizzi \& Bettuzzi, 2010; Rosenberg \& Silkensen, 1995; Shannan et al., 2006). This cytoprotective chaperoning role is particularly evident upon stress where sCLU increases the solubility of denatured proteins and mediates the uptake and degradation of a broad spectrum of hydrophobic denatured aggregated molecules (Poon, Treweek, Wilson, Easterbrook-Smith, \& Carver, 2002; Viard et al., 1999). Moreover, sCLU associates to, and stabilizes, the Ku70-Bax complex in the cytoplasm (Djeu \& Wei, 2009; Pucci et al., 2017) and is therefore believed to play an important role in survival and resistance to chemotherapeutic drugs (Koltai, 2014; Zhou et al., 2015). However, the elucidation of CLU's broad range of functions has been partly hampered by the finding that under cell stress or damage, a number of forms can be produced from the evolutionary conserved CLU gene either through alternative splicing/noncanonical translation/initiation start sites or eluding the glycosylation steps necessary for secretion (Bonacini et al., 2015; Prochnow et al., 2013). These intracellular forms display different intracellular localizations and, in some case, almost opposite biological functions (Al Nakouzi et al., 2016; July et al., 2002; Rizzi \& Bettuzzi, 2009; Rohne, Prochnow, \& Koch-Brandt, 2016).

Interestingly, CLU has been shown to affect the phosphatidylinositol 3'-kinase (PI3K)-AKT pathway (Ammar \& Closset, 2008; Jo, Jia, Subramanian, Hattori, \& Luo, 2008; Ma \& Bai, 2012). This pathway is the central hub of oncogenic signaling and has been linked to tumorigenesis in a wide variety of tumor types (Manning \& Toker, 2017; Ruzzene, Bertacchini, Toker, \& Marmiroli, 2017). In normal cells, stimulation of growth factor receptors activates acute production of phosphatidylinositol-3,4,5-triphosphate $\left(\mathrm{PIP}_{3}\right)$ by $\mathrm{PI} 3 \mathrm{~K}$, thus triggering dual AKT phosphorylation: at T308, by PDK1, and at S473, by mTORC2. Fully active AKT, in turn, enhances cell proliferation and survival (Fruman \& Rommel, 2014). Eventually, dephosphorylation of $\mathrm{PIP}_{3}$ by the lipid phosphatase PTEN and/or dephosphorylation of AKT S473 by PHLPP1/2 protein phosphatases turn off AKT signaling (Gao, Furnari, \&
Newton, 2005; Newton \& Trotman, 2014). Activation of PI3K/AKT signaling has been widely implicated in prostate cancer progression (Bertrand, McCubrey, Angus, Nutter, \& Sigounas, 2014; Manning \& Toker, 2017; McCubrey et al., 2015; Newton \& Trotman, 2014). Molecular alterations such as mutations, chromosomal aberrations, and altered expression of the key pathway components have been reported in $42 \%$ of primary and $100 \%$ of metastatic prostate cancers (Chen et al., 2011; Newton \& Trotman, 2014; Taylor et al., 2010). The majority of the above abnormalities include decreased expression of PTEN and/or of PHLPPs, therefore leading to constitutively active AKT signaling. Interestingly, PHLPP1 mRNA is downregulated in high-grade prostate cancer, suggesting that PHLPP1 loss contributes to other genetic aberrations to promote prostate cancer progression. Moreover, a study performed with AKT isoform-silencing showed that contrary to other models such as breast cancer cells, AKT1 and AKT2 cooperate to enhance the migration and invasion of PC3 cells through upregulation of $\beta 1$-integrin (Virtakoivu, Pellinen, Rantala, Perälä, \& Ivaska, 2012). On the other hand, AKT1 and AKT2 were described to play nonredundant functions in PC3 cell migration, possibly through distinct subcellular localization. Furthermore, it has been suggested that AKT isoformspecific contribution might differ during prostate cancer progression, from androgen-sensitive to hormone-resistant stage (Cariaga-Martinez et al., 2013). Therefore, mechanistically the peculiar roles of AKT isoforms in prostate cancer cell migration and invasion, if any, have not been conclusively settled. Here, we unravel a yet-undescribed regulatory system driven by SCLU, which modulates PHLPP1 level, leading to constitutive activation of AKT2 and enhanced migration and invasion of both normal and cancer prostate cells.

\section{2 | MATERIALS AND METHODS}

\subsection{Cell Cultures and Inhibitors}

Human prostate carcinoma PC3 cell line was obtained from deutsche sammlung von mikroorganismen und zellkulturen (DSMZ) (Braunschweig, Germany). Normal prostate epithelial PNT1A cells stably transfected with empty vector pIRES-hyg1 or pIRES-CLU were a kind gift from the laboratory of Dr. Saverio Bettuzzi (University of Parma, Italy; Scaltriti et al., 2004), and will hereafter be referred to as CLU and MOCK cells, respectively. PNT1A and PC3 cells were grown in RPMI 1640, Ham's F12 and Dulbecco's modified minimum essential medium (DMEM) supplemented with $1 \%$ L-glutamine, 10\% heat-inactivated fetal bovine serum (Euroclone, Milano, Italy), and antibiotics (SigmaAldrich, Darmstadt, Germany), at $37^{\circ} \mathrm{C}$ in a humidified atmosphere with 
$5 \% \mathrm{CO}_{2}$. Where indicated, the AKT2-selective inhibitor XII (Merck Millipore, Milano, Italy) was used at $0.5 \mu \mathrm{M}$.

The MTT assay was performed in 96-well plates. Ten thousand cells/well were seeded, and the cells were cultured for 24 and $48 \mathrm{hr}$. MTT (Sigma-Aldrich, Darmstadt, Germany) was dissolved in PBS at $5 \mathrm{mg} / \mathrm{ml}$, and $20 \mu \mathrm{l}$ of MTT solution was added to each well followed by $3 \mathrm{hr}$ of incubation at $37^{\circ} \mathrm{C}, 5 \% \mathrm{CO}_{2}$. Then, plates were read out by an ELISA reader (LifeTech, MA) at $590 \mathrm{~nm}$.

Cell proliferation was monitored by propidium iodide (PI) staining. After harvesting, the cells were washed twice with PBS and resuspended in $100 \mu \mathrm{l}$ binding buffer ( $10 \mathrm{mM} \mathrm{HEPES} / \mathrm{NaOH}$, of $\mathrm{pH} 7.4,140 \mathrm{mM} \mathrm{NaCl}$, $2.5 \mathrm{mM} \mathrm{CaCl}_{2}$ and $50 \mu \mathrm{g} / \mathrm{ml} \mathrm{PI}$ ). The samples were incubated for $10 \mathrm{~min}$ in the dark at $4{ }^{\circ} \mathrm{C}$ and then analyzed by flow cytometry.

\section{2 | Transfections}

For transient CLU overexpression, cells were transfected with polyethyleneimine (PEI) in a 3:1 ratio of $\mathrm{PEI}(\mu \mathrm{g})$ : total DNA $(\mu \mathrm{g})$. Control cells were transfected with empty vector. CLU silencing was performed with specific siRNA, and the cells were transfected for $72 \mathrm{hr}$ with siRNA transfection reagent as described in the supplier's instruction manual. For negative control, the cells were transfected with 100 nM scramble siRNA (all from Santa Cruz Biotechnology, Santa Cruz, CA). Where required, AKT1 and AKT2 were silenced with a set of AKT1 or AKT2 target shRNAs, kindly provided by the laboratory of Alex Toker (Beth Israel Deaconess Center, Boston). shAKT1 and shAKT2 were transduced in cells for $72 \mathrm{hr}$ by lentiviral supernatant obtained in packaging hosts HEK293 cells as described (Chin \& Toker, 2010). Infected cells were selected with $5 \mu \mathrm{g} / \mathrm{ml}$ of Puromycin for $48 \mathrm{hr}$.

\section{3 | Immunoblotting and Immunofluorescence}

Cell lysis was performed on ice for $20 \mathrm{~min}$ in a lysis buffer suitable for both reverse phase protein array (RPPA) and immunoblotting, as described previously (Maraldi et al., 2011). Briefly, for immunoblotting, lysates were sonicated three times for 10 seconds on ice, then centrifuged at $10,000 \mathrm{~g}$ for $15 \mathrm{~min}$ at $4^{\circ} \mathrm{C}$, and denatured at $100^{\circ} \mathrm{C}$ for $5 \mathrm{~min}$ in SDS-loading buffer, run on a 7\% SDS-PAGE, then transferred to an Immobilon-P PVDF membrane (Merck Millipore, Milano, Italy), and incubated overnight at $4^{\circ} \mathrm{C}$ with primary antibodies (Cenni et al., 2008). A list of antibodies is provided in Table 1S. For immunofluorescence, anti-PIP3 and anti-PIP2 antibodies (Echelon Biosciences, Salt Lake City, UT) were used at 1:50 dilution. The cells were analyzed using a Nikon Eclipse TE2000 inverted microscope, and fluorescent images were captured with a Hamamatsu camera (Hamamatsu City, Japan), as described previously (Cenni et al., 2014; Maraldi et al., 1993).

\section{4 | Reverse phase protein array}

For RPPA, protein lysates were diluted to a final concentration of $0.5 \mathrm{mg} / \mathrm{ml}$, and then $30 \mu \mathrm{l}$ were loaded on a 383-well plate and subjected to immunostaining with a panel of 82 commercially available, validated antibodies directed against specific phosphorylated or cleaved proteins, all from Cell Signaling Technology (Table 1S), as described (Bertacchini et al., 2014; Maraldi et al., 2011; Serafin et al., 2017).

\section{5 | RNA purification and real-time quantitative polymerase chain reaction}

Total RNA was extracted with the RNeasy Mini Kit (Qiagen, Valencia, CA) and reverse-transcribed with the High capacity cDNA Reverse Transcription kit (Applied Biosystem, Foster City, CA) according to the manufacturer's instructions. Quantification of miR-190 was performed with a miRCURY LNA Universal RT miR cDNA synthesis kit, SYBR Green master mix, Universal RT, and LNA PCR primer. Primer sequences are the following: PHLPP1, forward: 5'-TGCTCACT CCAACTGCATCGAG-3' reverse: 5'-GGTTTCCAGTCAGGTCTAGCT C-3'. miR-190, forward: 5'-TGATATGTTTGATATATTAGG-3' reverse: 5'-GAACATGTCTGCGTATCTC-3'. Glyceraldehyde 3-phosphate dehydrogenase, forward: 5'-CGAGATCCCTCCAAAATCAA-3' reverse: 5'-TTCACACCCATGACGAACAT-3'. The relative gene expression quantification was based on the comparative threshold cycle method $2^{-\Delta \Delta C_{t}}$. Raw data were normalized to the included housekeeping gene.

\subsection{Cell Migration and invasion assay}

Migration experiments were performed both by the transwell migration assay and by the XCELLigence RTCA System (CaRli biotech, Roma, Italy). For transwell migration experiments, $10^{5}$ cells were resuspended in $200 \mu \mathrm{l}$ of RPMI + BSA $3 \%$ and added to the upper chamber of a two-chamber device separated by the porous membrane (polycarbonate membrane inserts $8-\mu \mathrm{M}$ pore size; Corning, NY). The lower chambers were filled with $600 \mu \mathrm{l}$ of $\mathrm{NIH}$ 3T3 cells medium or with conditioned medium from CLU cells or with recombinant $\mathrm{CLU}$. The cells were incubated at $37^{\circ} \mathrm{C}$ for $48 \mathrm{hr}$ to allow cell migration through the membrane. Migratory cells were stained with the hematoxylin (HEMA) 3 STAIN kit (Thermo Scientific Fisher, Milano, Italy) and counted under a Nikon Eclipse TE2000 inverted microscope. Migration and invasion experiments with the xCELLigence RTCA System (Roche Applied Science, Mannheim, Germany) were performed following the manufacturer instructions, as described previously (Brugnoli et al., 2013; Grassilli et al., 2014). Briefly, $4 \times 10^{4}$ cells/well were seeded onto the top chambers of CIM-16 plates while the bottom chambers were filled with NIH-3T3 cells medium or with the CLU-conditioned medium. The signal detection was programmed every $15 \mathrm{~min}$ for a total of $48 \mathrm{hr}$. Impedance values were expressed as relative arbitrary units.

\section{7 | Statistical analysis}

Data were expressed as the mean \pm standard deviation (SD). Differences were tested by Student's $t$ test. The values $p<0.05$ were considered statistically significant, and were indicated by an asterisk *. The statistical analysis was done using the PRISM GraphPad statistical software. For RPPA, to detect differences between total protein 


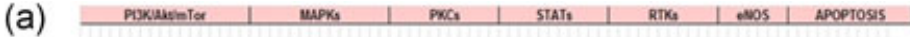

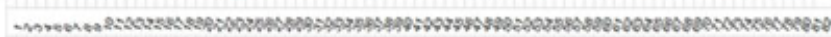

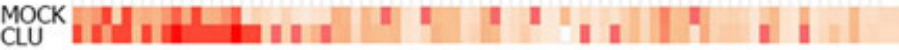

NIINSIrY

\section{1.}

(b)

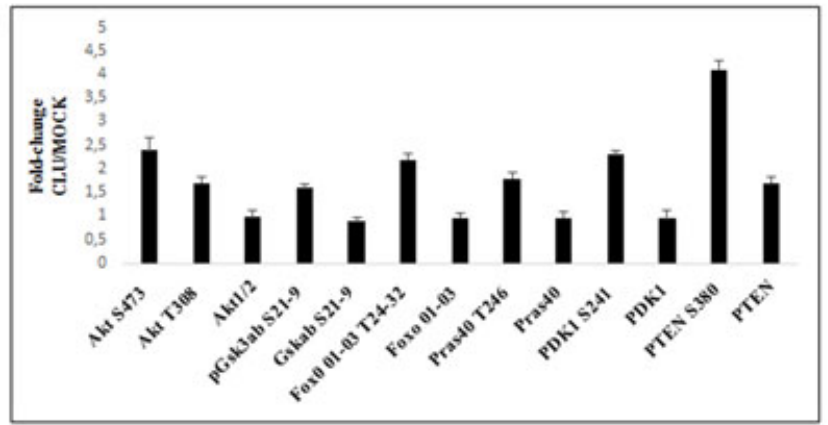

(d)

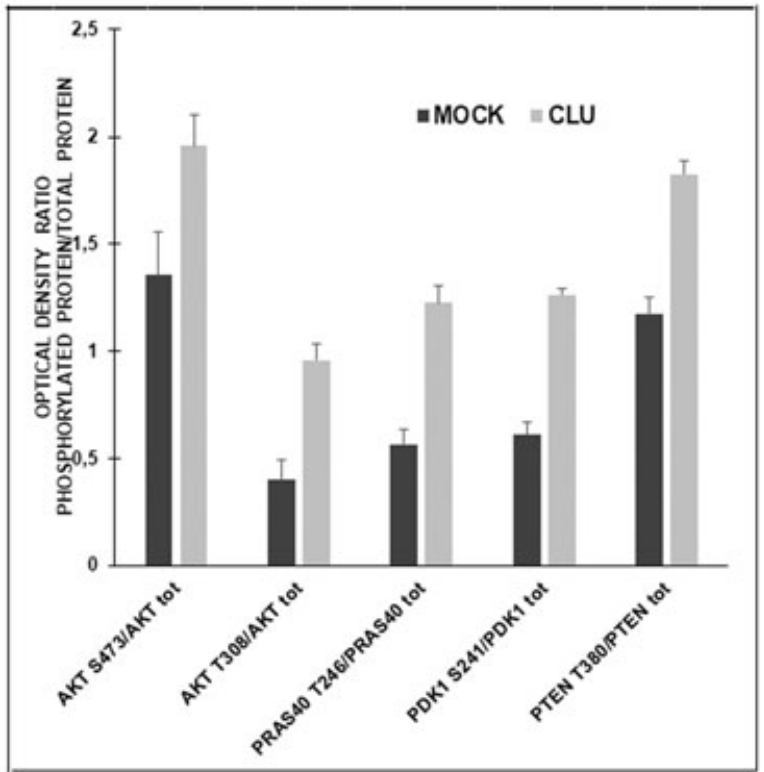

(c)

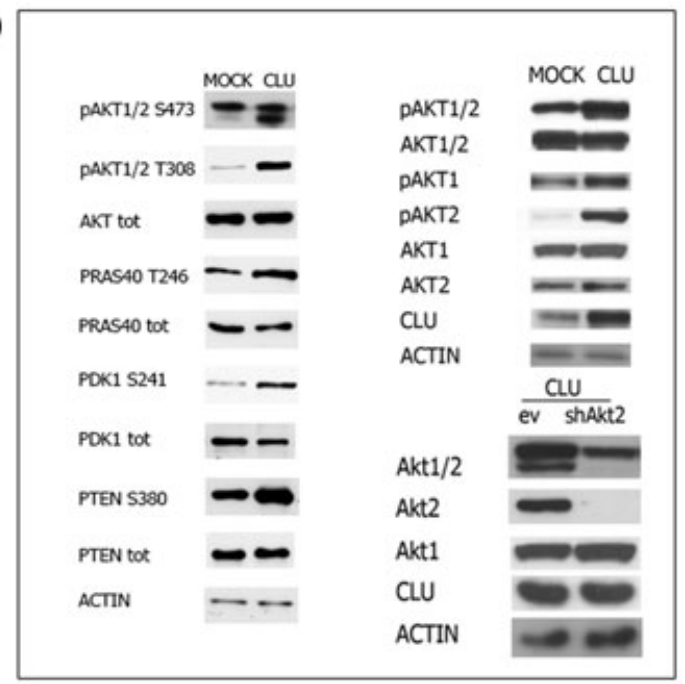

(e)

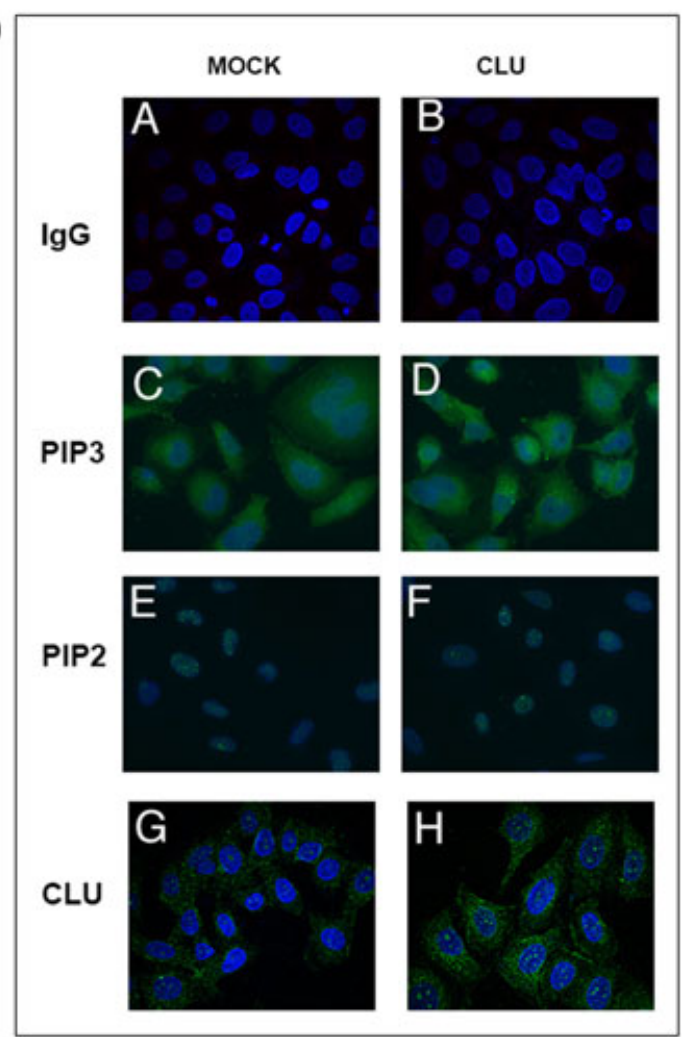

FIGURE 1 Clusterin enhances the PI3K signaling pathway at multiple levels. (a) Heatmap from the RPPA analysis of PNT1A cells stably transfected with empty vector (MOCK) or with clusterin (CLU). The colors in each cell indicate the signal intensities of single antibodies (RED, high intensity, WHITE low intensity). (b) Bar chart of key components of the PI3K/AKT cascade from (a). The X-axis reports all endpoints tested (phosphorylated epitopes where indicated); The $Y$-axis reports the fold-change ratio of signal intensity in CLU versus MOCK samples. The arbitrary unit of MOCK was set as 1, while the cutoff magnitude to qualify changes in specific proteins phosphorylation as significant was set as 1.2-fold. (c) Lysates from the above analysis were resolved by the Western blot analysis and probed with the indicated antibodies. Note that the anti-AKT S473 antibody detects both phosphorylated AKT1 S473 and AKT2 S474. The lower panel shows a comparison of AKT1 and two isoforms, both total and phosphorylated, in MOCK and CLU lysates by means of isoform-specific antibodies. The lower-right panel shows AKT2 and AKT1 silencing in CLU cells by isoform-specific shRNA. CLU cells transfected with scramble shRNA were run as a control. (d) Bar panel with quantitation of signal intensity of each band, normalized to the loading control, as the ratio of optical densities (OD) phosphorylated versus total protein. (e) PIP3, PIP2, and the CLU immunofluorescence analysis. Representative immunofluorescence photomicrographs of MOCK and CLU cells, showing PIP3, PIP2, and CLU content. Dilution: anti-PIP3 and anti-PIP2 (Echelon Laboratories), 1:50 dilution; anti-CLU, 1:100 (Millipore). Images were taken at a magnification of $\times 60$. PI3K, phosphatidylinositol 3'-kinase; PIP3, phosphatidylinositol-3,4,5-triphosphate; RPPA, reverse phase protein array [Color figure can be viewed at wileyonlinelibrary.com] 
expression and phosphorylated protein expression, a paired $t$ test was performed. We set 1.2-fold as the cutoff magnitude to qualify changes in specific proteins as significant.

\section{3 | RESULTS}

\subsection{CLU modulates the PI3K pathway}

MOCK and CLU cells were analyzed by RPPA to compare 82 native and phosphorylated proteins operating in key signaling pathways. Several endpoints were differentially expressed or phosphorylated (Figure 1a and Supporting Information Figure 1S). In particular, CLU cells showed a marked phosphorylation of proteins involved in the $\mathrm{PI3K} / \mathrm{AKT} / \mathrm{mTOR}$ cascade, such as PDK1, PTEN, as well as AKT itself and its direct substrates PRAS40 and Foxo (Figure 1b). This observation was confirmed by the Western blot analysis (Figure 1c). Although modulation of the AKT activity by CLU had been shown previously (Ammar \& Closset, 2008; Jo et al., 2008; Ma \& Bai, 2012), no molecular mechanism has been defined yet. We noticed that AKT phosphorylated at $\mathbf{S 4 7 3}$ migrated as a doublet (Figure 1c). Remarkably, while phosphorylation of the upper band was very similar in the two cell subtypes, the lower band was heavily phosphorylated in CLU cells but almost undetectable in MOCK cells. The antibody used detects AKT1, AKT2, and AKT3 phosphorylation at S473, S474, and S472, respectively, suggesting that the bands could correspond to different isoforms. However, in these cells, AKT3 is almost undetectable (not shown). By means of single isoform-silencing, the lower band proved to be AKT2, suggesting that CLU exerts an isoform-restricted control over AKT phosphorylation (Figure 1c, lower-right panel). This result, intriguing in itself, was even more so because by the same analysis, we found that the expression levels of the lipid phosphatase PTEN was almost doubled in CLU compared with MOCK cells. As PTEN dephosphorylates PIP3 (Maehama \& Dixon, 1998) and is therefore a well-known negative regulator of AKT (Milella et al., 2015; Myers et al., 1998; Song, Salmena, \& Pandolfi, 2012), this would suggest that a decrease, rather than an increase, of the AKT activity should occur. Nevertheless, along with the increased PTEN expression, PTEN phosphorylation at $\mathrm{S} 380$ was also altered, being dramatically higher in CLU cells (Figure 1b, c). Thus, because $\mathbf{S 3 8 0}$ phosphorylation is known to inhibit the PTEN phosphatase activity, while stabilizing the protein against degradation (Barata, 2011; Vazquez, Ramaswamy, Nakamura, \& Sellers, 2000), we concluded that the high level of pS380-PTEN in CLU cells actually contributes to AKT hyperactivation. To confirm this, we compared the content of PIP3 in MOCK and CLU cells by immunofluorescence. In good agreement with the above-mentioned results, the staining intensity of PIP3 was significantly higher in CLU cells with respect to the parental MOCK cells (Figure 1e). In contrast, PIP2 levels were similar, suggesting that the increased PIP3 level results more from PTEN downregulation than from PI3K activation (Figure 1e). Although high PIP3 levels can explain the sustained PDK1, S241, and AKT T308 phosphorylation, because the PI3K and PTEN activity were not measured directly, we cannot rule out that

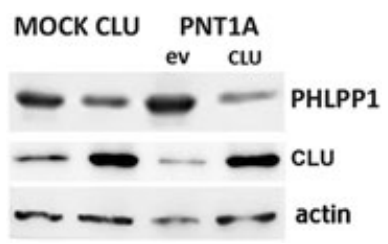

FIGURE 2 The analysis of PHLPP1 levels in CLU overexpressing cells. PHLPP1 was analyzed by the Western blot analysis in protein extracts from MOCK and CLU cells, and from PNT1A cells transfected with empty vector (ev) or with CLU for $24 \mathrm{hr}$, as indicated, then probed with anti-PHLPP1 from Bethyl Laboratories, 1:1,000. Lower panel, actin loading control. CLU, clusterin; PHLPP1, $\mathrm{PH}$ domain leucine-rich repeat-containing protein phosphatase 1

PIP3 derives from the PI3K activity rather than from PTEN inactivation. To assess whether the T308 phosphatase PP2A may also contribute to this mechanism, MOCK and CLU cells were incubated for 60 min with okadaic acid, a well-known PP2A inhibitor, and then the level of phospho-T308 in the two cell types was compared. In cells treated with okadaic acid and probed with antipT308, we observed a strong drop of the signal. However, it is also clear that the degree of inhibition of PP2A is the same in MOCK and CLU. Although not conclusive, this result suggests that the PP2A activity is similar in both cell lines (Figure 2).

Altogether, these findings could account for the 2.5-fold increase in total AKT phosphorylation detected by RPPA in CLU cells but could not explain the dramatic differential phosphorylation of AKT2S474 in CLU cells. We, therefore, decided to monitor the amount of the protein phosphatase specific to AKT2-S474, namely PHLPP1. We observed that PHLPP1 was markedly downregulated in CLU compared to MOCK cells (Figure 2), a condition that, by itself, could explain the AKT2 hyperactivity. PHLPP1 downmodulation was clearly detectable not only in cells stably expressing CLU but also in transiently transfected PNT1A cells, though to a lesser extent (Figure 2). These results prompted us to investigate the interplay between AKT2, PHLPP1, and CLU more deeply.

\subsection{CLU enhances miR-190, which, in turn, downmodulates PHLPP1}

From the RPPA analysis, it was evident that both the phosphorylation of GSK3 at its activatory residues and that of its main substrate $\beta$-catenin, at the GSK3 site S33/S37/T41, was more than doubled in CLU cells (Supporting Information Figure 2S). Interestingly, Li, Liu, and Gao (2009) demonstrated that PHLPP1 is a proteolytic target of the E3 ligase $\beta$-TrCP and undergoes proteasomal degradation upon sequential phosphorylation by CK1 and GSK3. This suggested that CLU might promote GSK3-mediated PHLPP1 degradation. Moreover, we also analyzed the effect of CLU overexpression on PHLPP1 expression. Interestingly, we found that PHLPP1 mRNA was dramatically downregulated (>10-fold) in PNT1A cells transfected with CLU, compared with empty vector-transfected controls (Figure 3a, left). Conversely, silencing of endogenous CLU by specific siRNA triggered a 6- to 8-fold increase in PHLPP1 mRNA, clearly indicating that CLU indeed modulates its 
(a)

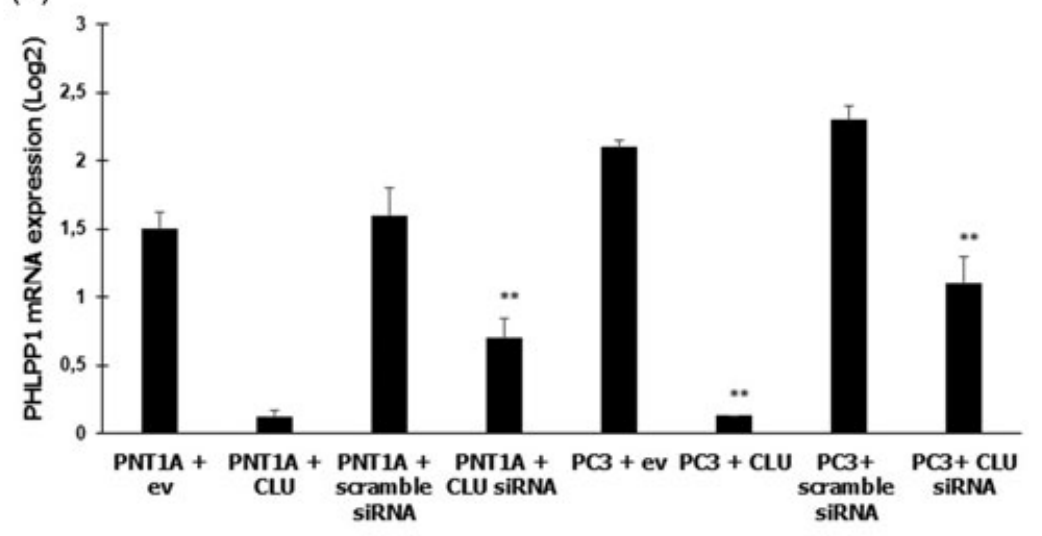

(b)

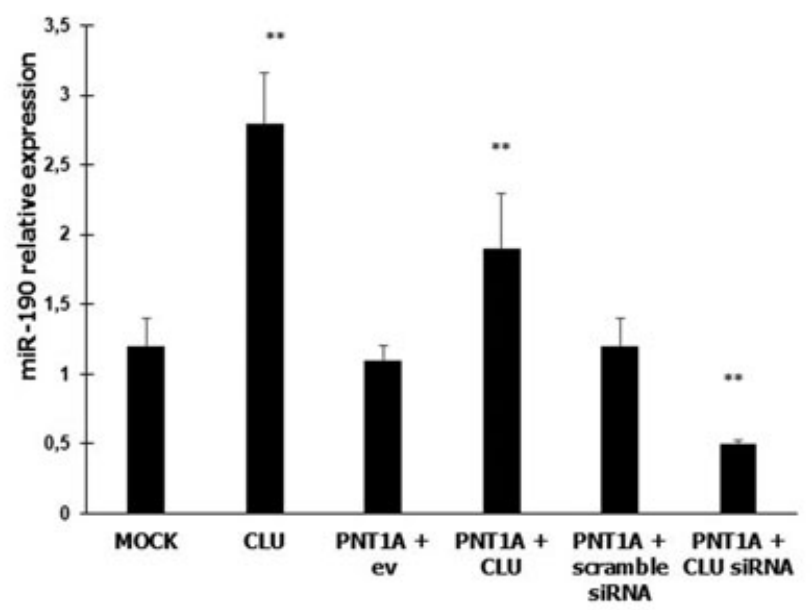

(c)

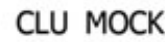
PNT1A $+e v+$ CLU + scr +siRNA

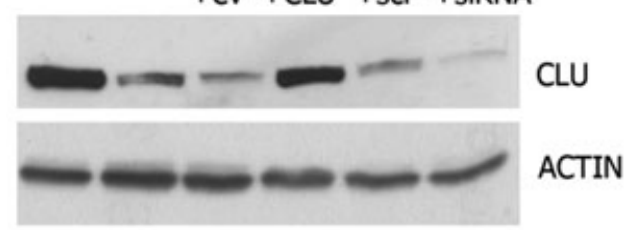

$\mathrm{PC} 3$

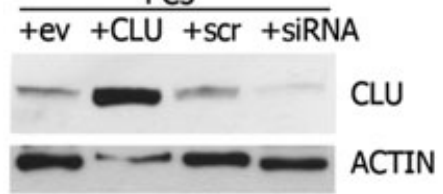

FIGURE 3 Modulation of PHLPP1 expression by CLU. (a) The RT-PCR analysis of PHLPP1 mRNA in PNT1A and PC3 cells. CLU was either overexpressed (CLU) or silenced with specific siRNA (siRNA) by transfection for $72 \mathrm{hr}$. Control cells were transfected with empty vector (ev) or with a scrambled sequence (scramble). ${ }^{* *} p<0.05$. (b) miR-190 levels were determined by RT-PCR in MOCK, CLU, and PNT1A cells from (a). (c) CLU expression was probed in all the above experimental points by the anticlusterin Western blot analysis followed by antiactin control of protein loading. Results represent the means from three independent experiments $\pm S D$. CLU, clusterin; PHLPP1, PH domain leucine-rich repeat-containing protein phosphatase 1; RT-PCR, real-time polymerase chain reaction; $S D$, standard deviation transcription. As PNT1A are normal prostatic epithelial cells, we wondered whether CLU might display the same effects also in prostate cancer cells, such as a highly tumorigenic PC3 cell line. We found that the expression of PHLPP1 was unquestionably upregulated by CLU silencing (>20-fold) and downmodulated by overexpression, with a trend similar to that observed in PNT1A cells, although to a greater extent (Figure 3a, right). Recently, a number of miRs have been shown to control the expression of PHLPPs (Grzechnik \& Newton, 2016). In particular, miR190 and miR-214 have been shown by us and others to regulate PHLPPs expression in prostate epithelial cells (Beezhold et al., 2011). Thus, we asked whether CLU might tune PHLPP1 levels through such molecules. The expression of both miR-190 and miR-214 was analyzed by real-time polymerase chain reaction. While the level of miR-214 was not affected (not shown), that of miR-190 was significantly higher in CLU compared with MOCK cells, and a similar increase was observed upon the forced expression of CLU, particularly in PC3 cells (39-fold increase; Figure 3b). Thus, we conclude that CLU exerts a complex control of PHLPP1 phosphatase regulation mediated by an epigenetic mechanism involving increased expression of miR-190, which eventually leads to constitutive phosphorylation of AKT2. 


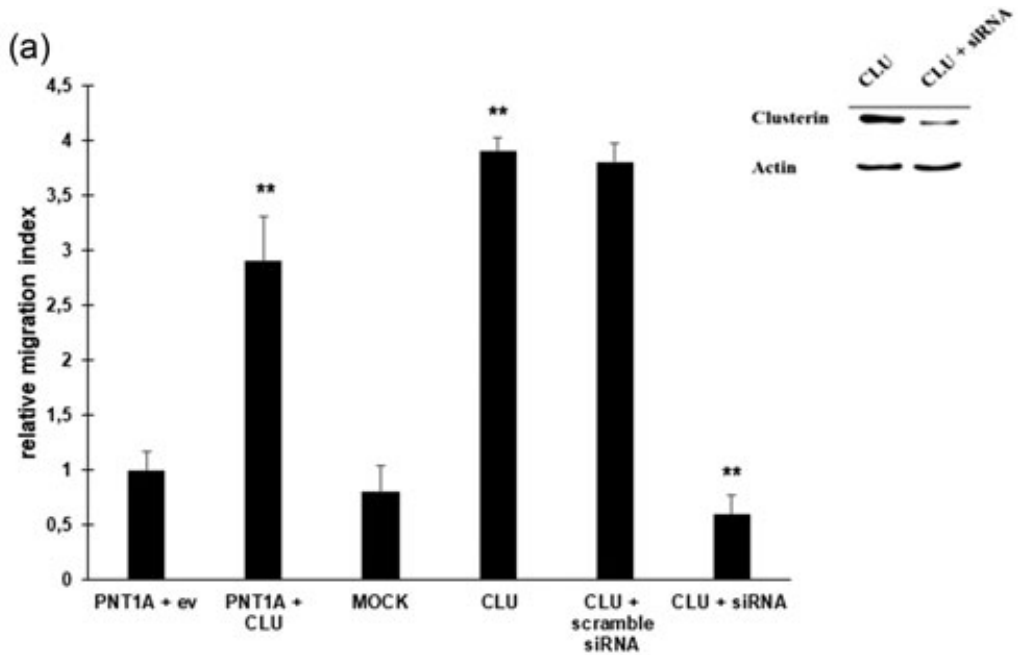

(b)
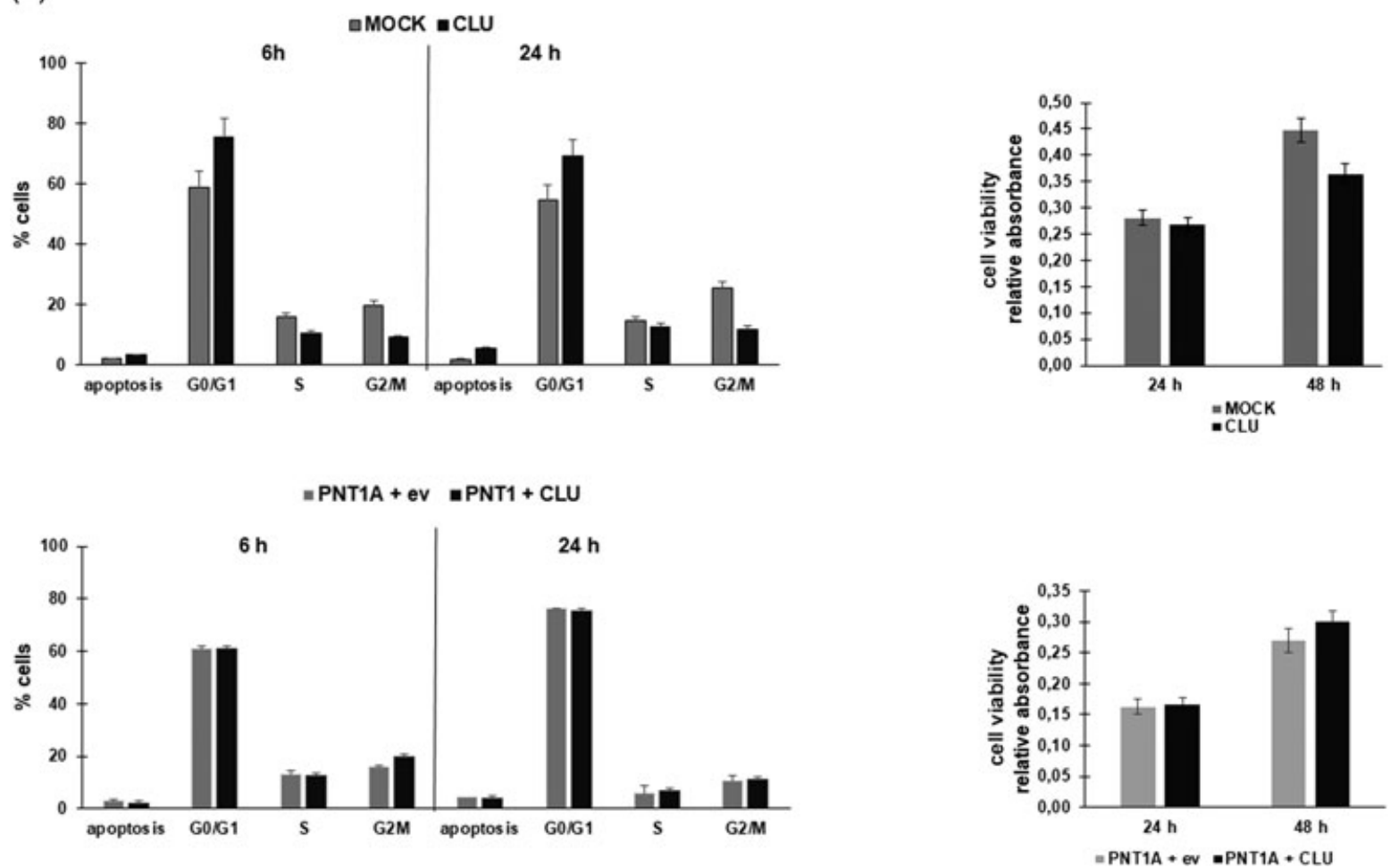

FIGURE 4 CLU stimulates migration of normal prostate cells. (a) Bar graph representation of the cell migration analysis by the transwell assay. Aliquots of the cells from the experimental points described in Figure 3a,b were seeded on transwells to analyze cell migration. Relative migration was quantified by counting the number of violet and blue cells (HEMA staining) in three inserts. At least three different experiments were performed. Results are average $\pm S D$. Silencing of CLU in CLU cells by transfection with specific siRNA for $72 \mathrm{hr}$ (which were not included in the experimental set above) was confirmed by the Western blot analysis (figure [a] insert). Control CLU cells were transfected with a scramble sequence. (b) To verify whether modulation of CLU expression affects cell proliferation or cell viability, aliquots of cells from the above experimental points were analyzed for the cell cycle progression by PI staining followed by flow cytometry (left panels) and for cell proliferation by the MTT assay (right panels). CLU, clusterin; MTT, 3-(4,5-dimethylthiazol-2-yl)-2,5-diphenyltetrazolium; SD, standard deviation

\section{3 | CLU facilitates cell migration and invasion through a PHLPP1-AKT2 circuit}

Overexpression of CLU has been reported to enhance the metastatic behavior of human laryngeal squamous carcinoma cells (Wang, Cao, Su, Liu, \& Zhang, 2014), as well as renal
(Shi et al., 2013; Wang, Luo, Dong, Yu, \& Zhao, 2014), breast (Niu et al., 2012), and hepatocellular carcinoma (Wang et al., 2012). Conversely, CLU was demonstrated to inhibit prostate cancer cell migration by associating to $\alpha$-actinin and disassembling of the actin cytoskeleton (Moretti et al., 2007; Rizzi \& Bettuzzi, 2010). Our results clearly indicated that CLU stable clone cells 
exhibited, over a 24-hr time frame, a migration index more than three-fold higher than their MOCK counterpart. On the contrary, silencing of CLU by specific siRNA strongly inhibited migration of CLU cells (Figure 4a). Therefore, we analyzed the effects of CLU transient overexpression on the migration of PNT1A cells, which are not expected to display a high mobility phenotype. Remarkably, transient transfection of PNT1A cells for $48 \mathrm{hr}$ was sufficient to boost their migration almost to the levels of CLU cells (Figure 4a). As the transwell assay used to evaluate migration did not detect alterations of cell proliferation or cell viability, the possibility that such effects might be evoked by CLU during the 48-hr time frame were ruled out by means of the analysis of the cell cycle through PI staining and flow cytometry as well as of the viability by the MTT assay (Figure 4b).

Next, we explored the effect of AKT silencing on migration of MOCK versus CLU cells. We found that while AKT1 silencing affected migration of neither MOCK nor CLU cells, silencing of AKT2 prevented migration of both cell types (Figure 5a). Treatment with the AKT2-selective pharmacological inhibitor AKT Inhibitor XII (Bulj et al., 2013; Zhao et al., 2008) at a dose as low as $500 \mathrm{nM}$, gave similar results (Figure $5 \mathrm{c}$ ). Altogether, these findings indicate that CLU likely controls prostate epithelial cell migration predominantly through downregulation of PHLPP1 and activation of AKT2.

Next, we investigated how CLU might affect the movement of prostate cancer cells with an invasive phenotype. Thus, the migration capability of PNT1A cells was compared with that of PC 3 cells, which display increased invasiveness, upon transfection with either empty vector or with CLU. As expected, PC3 cells displayed a higher basal migration index than PNT1A, which was nevertheless dramatically increased by overexpression of CLU in both cell types (Figure 6a). What's more important, we further evaluated the effects of CLU expression in cells grown in a 3D matrix. The results are clear and show that CLU overexpression significantly stimulated cell invasion through Matrigel (Figure 6b). These results are in complete agreement with the observation that the expression of CLU increases, whereas its silencing decreases,

(a)

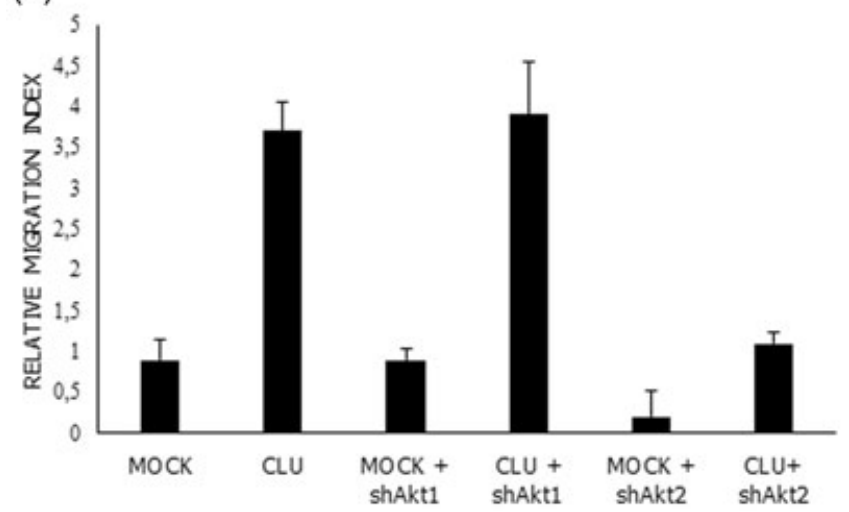

(b)

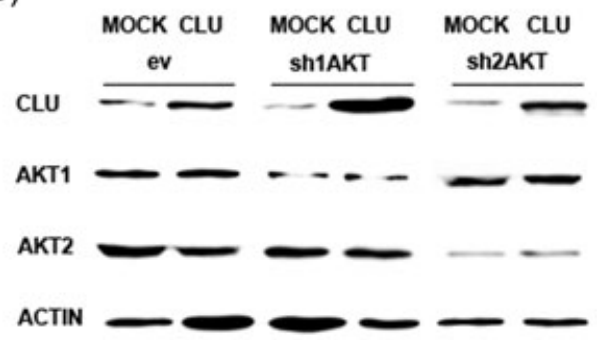

(c)

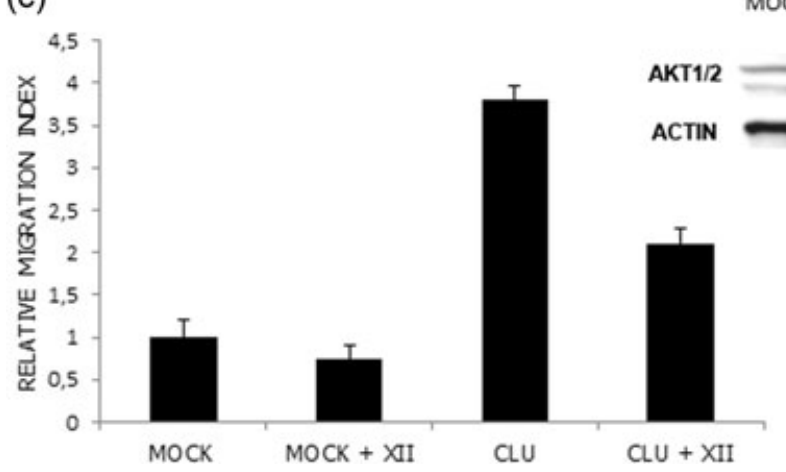

FIGURE 5 Effect of AKT isoformsilencing on CLU-stimulated cell migration. (a) The bar graph shows the relative migration of MOCK and CLU cells after AKT1 or AKT2 silencing with isoformspecific shRNAs. Migration was quantified by HEMA staining in three inserts, as described above. (b) Samples from each experimental point were resolved by the Western blot analysis and probed with isoform-specific antibodies to confirm silencing. (c) Relative migration of $\mathrm{MOCK}$ and CLU cells $48 \mathrm{hr}$ upon addition to the growth medium of the specific AKT2 inhibitor (XII) at $0.5 \mu \mathrm{M}$. Relative migration was quantified as described above. (d) AKT2 inhibition was confirmed by the Western blot analysis with anti-pAKT1/2. At least three different migration experiments were performed. Results are average $\pm S D$. CLU, clusterin; $S D$, standard deviation 


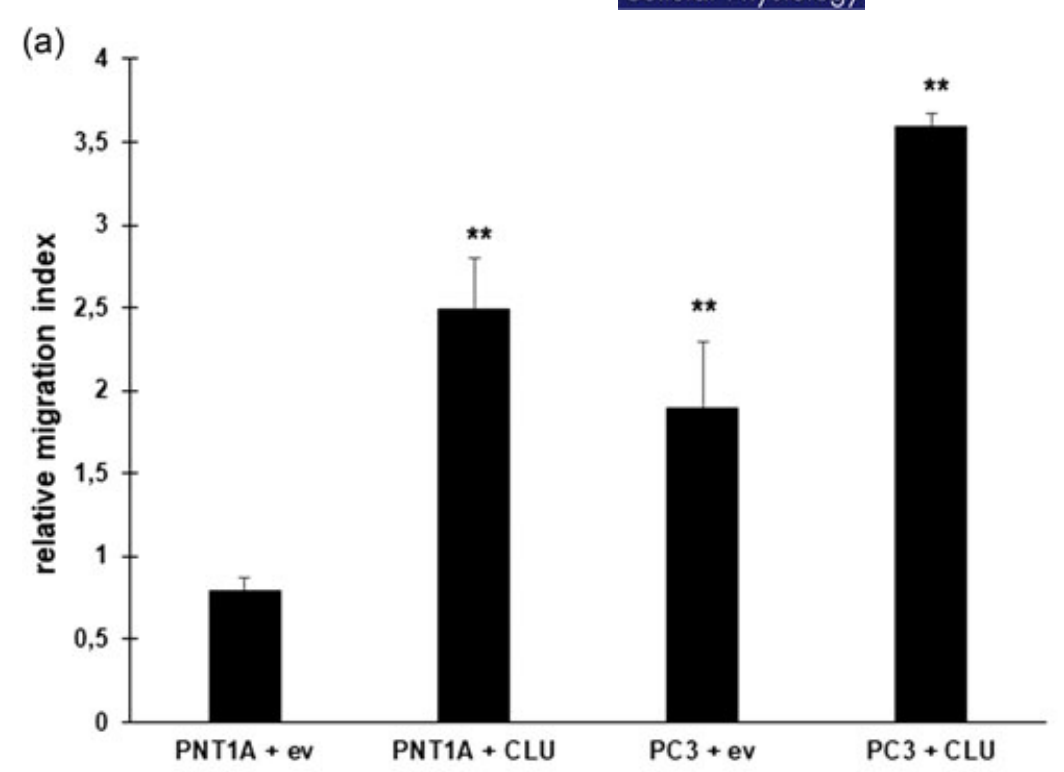

FIGURE 6 Modulation of normal and cancer cell motility by CLU. PNT1A and PC3 cells from the same pool analyzed in Figure 3 (transfected with empty vector or with CLU) were run with the xCELLigence System to evaluate the modulation of migration (a) and invasion (b) by CLU. The relative migration index is indicated in the $Y$-axis. Migration and invasion experiments were repeated two times. Results are average $\pm S D$. CLU, clusterin; $S D$, standard deviation (b)

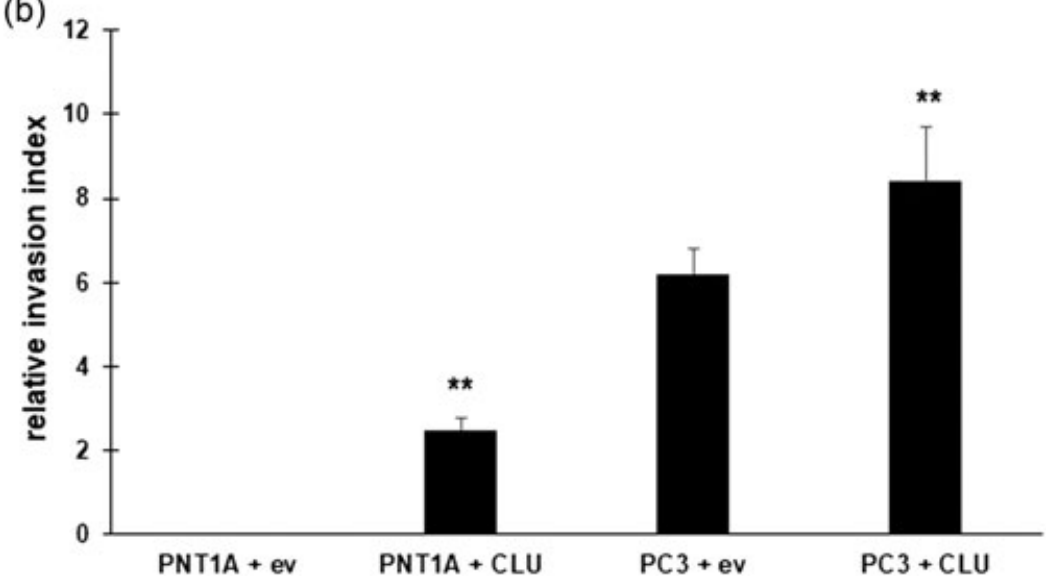

PHLPP1 expression in both PNT1A and PC3 cells, while inversely affecting MiR-190. These results altogether conclude that CLU exerts a migration and proinvasive effect on healthy epithelial prostate cells through a miR-190/PHLPP1/AKT 2 circuit.

\section{4 | DISCUSSION}

CLU is a small heat-shocklike protein overexpressed in many solid tumors with multiple functions but largely elusive molecular mechanisms. Work from independent laboratories has suggested that it can modulate the PI3K/AKT pathway, although in a cell-type and contextdependent manner. In particular, CLU has been shown to block the PI3K activity through the association with insuline-like growth factor (IGF-1) and inhibition of IGF-1R during serum deprivation (Jo et al., 2008), to mediate IGF-1 induced activation of PI3K signaling in nonsmall-cell lung cancer (Ma \& Bai, 2012) or to activate AKT and promote the survival of prostate cancer cells (Ammar \& Closset, 2008). On the other hand, AKT has been shown to upregulate SCLU which, in turn, would mediate docetaxel resistance in prostate cancer cells (Zhong et al., 2010). By a wide analysis of the phosphoproteome of normal prostate PNT1A cells overexpressing CLU, the first part of our study shows that CLU impinges on the PI3K/AKT cascade at multiple levels and, by doing so, hyperactivates AKT. The enhanced phosphorylation of AKT at T308 could be explained by our finding that in CLU cells, the specific PIP3 phosphatase PTEN (Maehama \& Dixon, 1998) is highly phosphorylated at the inhibitory site T380 (Vazquez et al., 2000). However, we unambiguously demonstrate for the first time that CLU also evokes a dramatic differential phosphorylation of AKT2. Although the parallel inactivation of PTEN can undeniably contribute to AKT2 activation, it cannot account for the observed isoform selectivity. Taking advantage of the notion that the phosphatases, that dephosphorylate AKT at S473, display an isoform-specific activity (Gao et al., 2005; Newton \& Trotman, 2014), we disclose here that the AKT2-specific phosphatase, PHLPP1, is strongly downregulated in CLU cells. PHLPP1 has been reported to undergo proteasome-dependent degradation after phosphorylation by GSK3 and CK1 in colon cancer cells (Li et al., 2009). While on the one hand, our results suggest that CLU overexpression might in part promote such mechanism also in normal prostate cells, on the other hand, we have clearly demonstrated that CLU mainly acts by preventing mRNA translation of PHLPP1. Importantly, because it has been recently demonstrated that PHLPP expression can be modulated 
through specific miRs (Beezhold et al., 2011), and in particular in prostate cancer cells by the upregulation of miR-190 and miR-214, we investigated whether CLU alters the expression of these molecules. Although we did not observe any change in miR-214 level, we were able to show that the intracellular content of miR-190 in cells either transfected with or exposed to CLU is indeed affected to a very large extent (up to a 39-fold increase in PC3 cells). An opposite result was obtained by CLU silencing. In light of the effects of CLU on PHLPP1 expression and the resulting peculiar phosphorylation pattern of AKT isoforms, we assessed whether CLU might affect cell motility. A pioneering work by Scaltriti et al. (2004) suggested that CLU counteracts cell migration and invasion. Conversely, more recent works from different laboratories indicate that it enhances cell migration in several cell models (Jin et al., 2012; Niu et al., 2012). Although there is some evidence that in macrophages, CLU promotes chemotactic migration through upregulation of matrix metalloproteinase- 9 and NF-kB activation downstream of AKT and Erk1/2 (Shim et al., 2011; Shim, Kang, Choi, Park, \& Min, 2012), hitherto its molecular mechanisms in prostate cells have remained elusive. We show that overexpression of CLU triggers migration both in normal and in cancer prostate cells, and is sufficient to increase the migration index of normal PNT1A cells to the same level of the highly metastatic PC3 prostate cancer cells. Conversely, CLU silencing prevents migration in both cell types. In particular, on the basis of the effects of specific silencing of either AKT1 or AKT2, we further demonstrate that CLU drives cell migration through AKT2. Inhibition of the AKT2 activity by the isoform-specific allosteric inhibitor AKTi XII supported this conclusion. A CLU-induced decrease in PHLPP1 expression is in complete agreement with this function of AKT2. Moreover, we demonstrate that CLU overexpression is sufficient to boost PC3 cell invasion through a 3D Matrigel, whereas CLU silencing completely abrogates it.

Thus, all together, our results clearly demonstrate for the first time that the CLU promigration activity is controlled by the PHLPP/ AKT axis, as CLU can switch the low migration and invasion phenotype of normal prostate cells toward the high migration phenotype typical of cancer PC3 cells through the modulation of the expression of PHLPP1 and, in turn, the activity of AKT2.

\section{ACKNOWLEDGMENTS}

The authors thank the laboratory of Dr. Bettuzzi for providing MOCK and CLU clones, as well as CLU expression vector. This study was supported by grants from Fondazione Cassa Risparmio Vignola to SM.

\section{CONFLICTS OF INTEREST}

The authors declare that there are no conflicts of interest.

\section{ORCID}

Jessika Bertacchini (D) http://orcid.org/0000-0001-9218-9215 Lucio Cocco (D) http://orcid.org/0000-0002-9206-8277 Sandra Marmiroli (D) http://orcid.org/0000-0001-5545-9319

\section{REFERENCES}

Al Nakouzi, N., Wang, C. K., Beraldi, E., Jager, W., Ettinger, S., Fazli, L., ... Gleave, M. (2016). Clusterin knockdown sensitizes prostate cancer cells to taxane by modulating mitosis. EMBO Molecular Medicine, 8 , 761-778.

Ammar, H., \& Closset, J. L. (2008). Clusterin activates survival through the phosphatidylinositol 3-kinase/Akt pathway. Journal of Biological Chemistry, 283, 12851-12861.

Barata, J. T. (2011). The impact of PTEN regulation by CK2 on PI3Kdependent signaling and leukemia cell survival. Advances in Enzyme Regulation, 51, 37-49.

Beezhold, K., Liu, J., Kan, H., Meighan, T., Castranova, V., Shi, X., \& Chen, F. (2011). miR-190-mediated downregulation of PHLPP contributes to arsenic-induced Akt activation and carcinogenesis. Toxicological Sciences, 123, 411-420.

Bertacchini, J., Guida, M., Accordi, B., Mediani, L., Martelli, A. M., Barozzi, P., ... Marmiroli, S. (2014). Feedbacks and adaptive capabilities of the PI3K/ $\mathrm{Akt} / \mathrm{mTOR}$ axis in acute myeloid leukemia revealed by pathway selective inhibition and phosphoproteome analysis. Leukemia, 28, 2197-2205.

Bertrand, F. E., McCubrey, J. A., Angus, C. W., Nutter, J. M., \& Sigounas, G. (2014). NOTCH and PTEN in prostate cancer. Advances in Biological Regulation, 56, 51-65.

Bonacini, M., Coletta, M., Ramazzina, I., Naponelli, V., Modernelli, A., Davalli, P., ... Rizzi, F. (2015). Distinct promoters, subjected to epigenetic regulation, drive the expression of two clusterin mRNAs in prostate cancer cells. Biochimica et Biophysica Acta/General Subjects, 1849, 44-54.

Brugnoli, F., Grassilli, S., Piazzi, M., Palomba, M., Nika, E., Bavelloni, A., ... Bertagnolo, V. (2013). In triple negative breast tumor cells, PLC- $\beta 2$ promotes the conversion of CD133high to CD133low phenotype and reduces the CD133-related invasiveness. Molecular Cancer, 12, 165-180.

Bulj, Z., Duchi, S., Bevilacqua, A., Gherardi, A., Dozza, B., Piccinini, F., ... Marmiroli, S. (2013). Protein kinase B/AKT isoform 2 drives migration of human mesenchymal stem cells. International Journal of Oncology, 42, 118-126.

Cariaga-Martinez, A. E., López-Ruiz, P., Nombela-Blanco, M. P., Motiño, O., González-Corpas, A., Rodriguez-Ubreva, J., ... Colás, B. (2013). Distinct and specific roles of AKT1 and AKT2 in androgen-sensitive and androgen-independent prostate cancer cells. Cellular Signalling, 25, 1586-1597.

Cenni, V., Bertacchini, J., Beretti, F., Lattanzi, G., Bavelloni, A., Riccio, M., ... Marmiroli, S. (2008). Lamin A Ser404 is a nuclear target of Akt phosphorylation in $\mathrm{C} 2 \mathrm{C} 12$ cells. Journal of Proteome Research, 7 , 4727-4735.

Cenni, V., Capanni, C., Mattioli, E., Columbaro, M., Wehnert, M., Ortolani, M., ... Lattanzi, G. (2014). Rapamycin treatment of Mandibuloacral dysplasia cells rescues localization of chromatin-associated proteins and cell cycle dynamics. Aging (Albany NY), 6, 755-770.

Chen, M., Pratt, C. P., Zeeman, M. E., Schultz, N., Taylor, B. S., O'Neill, A., ... Trotman, L. C. (2011). Identification of PHLPP1 as a tumor suppressor reveals the role of feedback activation in PTEN-mutant prostate cancer progression. Cancer Cell, 20, 173-186.

Chin, Y. R., \& Toker, A. (2010). The actin-bundling protein palladin is an Akt1-specific substrate hat regulates breast cancer cell migration. Molecular Cell, 38, 333-344.

Choi-Miura, N. H., Takahashi, Y., Nakano, Y., Tobe, T., \& Tomita, M. (1992). Identification of the disulfide bonds in human plasma protein SP-40 (apolipoprotein-J). Journal of Biochemistry, 112, 557-561.

Djeu, J. Y., \& Wei, S. (2009). Clusterin and chemoresistance. Advances in Cancer Research, 105, 77-92.

Fruman, D. A., \& Rommel, C. (2014). PI3K and cancer: Lessons, challenges and opportunities. Nature reviews. Drug Discovery, 13, 140-156. 
Gao, T., Furnari, F., \& Newton, A. C. (2005). PHLPP: A phosphatase that directly dephosphorylates Akt, promotes apoptosis, and suppresses tumor growth. Molecular Cell, 18, 13-24.

Grassilli, S., Brugnoli, F., Lattanzio, R., Rossi, C., Perracchio, L., Mottolese, M., ... Bertagnolo, V. (2014). High nuclear level of Vav1 is a positive prognostic factor in early invasive breast tumors: A role in modulating genes related to the efficiency of metastatic process. Oncotarget, 5, 4320-4336.

Grzechnik, A. T., \& Newton, A. C. (2016). PHLPPing through history: A decade in the life of PHLPP phosphatases. Biochemical Society Transactions, 44, 1675-1682.

Jin, J., Kim, J. M., Hur, Y. S., Cho, W., Lee, K. Y., Ahn, S. I., ... Park, I. S. (2012). Clinical significance of clusterin expression in pancreatic adenocarcinoma. World Journal of Surgical Oncology, 10, 146-151.

Jo, H., Jia, Y., Subramanian, K. K., Hattori, H., \& Luo, H. R. (2008). Cancer cell-derived clusterin modulates the phosphatidylinositol 3'-kinase-Akt pathway through attenuation of insulin-like growth factor 1 during serum deprivation. Molecular and Cellular Biology, 28, 4285-4299.

July, L. V., Akbari, M., Zellweger, T., Jones, E. C., Goldenberg, S. L., \& Gleave, M. E. (2002). Clusterin expression is significantly enhanced in prostate cancer cells following androgen withdrawal therapy. Prostate, 50, 179-188.

Kim, N., \& Choi, W. S. (2011). Proapoptotic role of nuclear clusterin in brain. Anatomy \& Cell Biology, 44, 169-175.

Koltai, T. (2014). Clusterin: A key player in cancer chemoresistance and its inhibition. OncoTargets and Therapy, 7, 447-456.

Leskov, K. S., Klokov, D. Y., Li, J., Kinsella, T. J., \& Boothman, D. A. (2003). Synthesis and functional analyses of nuclear clusterin, a cell death protein. Journal of Biological Chemistry, 278, 11590-11600.

Li, X., Liu, J., \& Gao, T. (2009). $\beta$-TrCP-mediated ubiquitination and degradation of PHLPP1 are negatively regulated by Akt. Molecular and Cellular Biology, 29, 6192-6205.

Ma, X., \& Bai, Y. (2012). IGF-1 activates the P13K/AKT signaling pathway via upregulation of secretory clusterin. Molecular Medicine Reports, 6 , 1433-1437.

Maehama, T., \& Dixon, J. E. (1998). The tumor suppressor, PTEN/MMAC1, dephosphorylates the lipid second messenger, phosphatidylinositol 3,4,5-trisphosphate. Journal of Biological Chemistry, 73, 13375-13378.

Manning, B. D., \& Toker, A. (2017). AKT/PKB Signaling: Navigating the network. Cell, 169, 381-405.

Maraldi, N. M., Zini, N., Santi, S., Bavelloni, A., Valmori, A., Marmiroli, S., \& Ognibene, A. (1993). Phosphoinositidase $C$ isozymes in SaOS-2 cells: Immunocytochemical detection in nuclear and cytoplasmic compartments. Biology of the Cell, 79, 243-250.

Maraldi, T., Bertacchini, J., Benincasa, M., Guida, M., De Pol, A., Liotta, L. A., ... Marmiroli, S. (2011). Reverse-phase protein microarrays (RPPA) as a diagnostic and therapeutic guide in multidrug resistant leukemia. International Journal of Oncology, 38, 427-435.

McCubrey, J. A., Abrams, S. L., Fitzgerald, T. L., Cocco, L., Martelli, A. M., Montalto, G., ... Steelman, L. S. (2015). Roles of signaling pathways in drug resistance, cancer initiating cells and cancer progression and metastasis. Advances in Biological Regulation, 57, 75-101.

Milella, M., Falcone, I., Conciatori, F., Cesta Incani, U., Del Curatolo, A., Inzerilli, N., ... Ciuffreda, L. (2015). PTEN: Multiple functions in human malignant tumors. Frontiers in Oncology, 5, 24-38.

Moretti, R. M., Marelli, M. M., Mai, S., Cariboni, A., Scaltriti, M., Bettuzzi, S., \& Limonta, P. (2007). Clusterin isoforms differentially affect growth and motility of prostate cells: Possible implications in prostate tumorigenesis. Cancer Research, 67, 10325-10333.

Myers, M. P., Pass, I., Batty, I. H., Van der Kaay, J., Stolarov, J. P., Hemmings, B. A., ... Tonks, N. K. (1998). The lipid phosphatase activity of PTEN is critical for its tumor supressor function. Proceedings of the National Academy of Sciences of the United States of America, 95, 13513-13518.
Newton, A. C., \& Trotman, L. C. (2014). Turning off AKT: PHLPP as a drug target. Annual Review of Pharmacology and Toxicology, 54, 537-558.

Niu, Z., Li, X., Hu, B., Li, R., Wang, L., Wu, L., \& Wang, X. (2012). Small interfering RNA targeted to secretory clusterin blocks tumor growth, motility, and invasion in breast cancer. Acta biochimica et biophysica Sinica, 44, 991-998.

Poon, S., Treweek, T. M., Wilson, M. R., Easterbrook-Smith, S. B., \& Carver, J. A. (2002). Clusterin is an extracellular chaperone that specifically interacts with slowly aggregating proteins on their off-folding pathway. FEBS Letters, 513, 259-266.

Prochnow, H., Gollan, R., Rohne, P., Hassemer, M., Koch-Brandt, C., \& Baiersdörfer, M. (2013). Non-secreted clusterin isoforms are translated in rare amounts from distinct human mRNA variants and do not affect Bax-mediated apoptosis or the NF-kB signaling pathway. PLoS One, 8, e75303.

Pucci, S., Mazzarelli, P., Nucci, C., Ricci, F., \& Spagnoli, L. G. (2009). CLU "in and out": Looking for a link. Advances in Cancer Research, 105, 93-113.

Pucci, S., Polidoro, C., Joubert, A., Mastrangeli, F., Tolu, B., Benassi, M., ... Santoni, R. (2017). Ku70, Ku80, and sClusterin: A cluster of predicting factors for response to neoadjuvant chemoradiation therapy in patients with locally advanced rectal cancer. International Journal of Radiation Oncology, Biology, Physics (Elmsford, NY), 97, 381-388.

Rizzi, F., \& Bettuzzi, S. (2009). Clusterin (CLU) and prostate cancer. Advances in Cancer Research, 105, 1-19.

Rizzi, F., \& Bettuzzi, S. (2010). The clusterin paradigm in prostate and breast carcinogenesis. Endocrine-related Cancer, 17, R1-R17.

Rohne, P., Prochnow, H., \& Koch-Brandt, C. (2016). The CLU-files: Disentanglement of a mystery. BioMol Concepts, 7, 1-15.

Rosenberg, M. E., \& Silkensen, J. (1995). Clusterin: Physiologic and pathophysiologic considerations. International Journal of Biochemistry \& Cell Biology, 27, 633-645.

Ruzzene, M., Bertacchini, J., Toker, A., \& Marmiroli, S. (2017). Cross-talk between the CK2 and AKT signaling pathways in cancer. Adv Biol Regul, 64, 1-8.

Scaltriti, M., Bettuzzi, S., Sharrard, R. M., Caporali, A., Caccamo, A. E., \& Maitland, N. J. (2004). Clusterin overexpression in both malignant and nonmalignant prostate epithelial cells induces cell cycle arrest and apoptosis. British Journal of Cancer, 91, 1842-1850.

Serafin, V., Lissandron, V., Buldini, B., Bresolin, S., Paganin, M., Grillo, F., ... Accordi, B. (2017). Phosphoproteomic analysis reveals hyperactivation of mTOR/STAT3 and LCK/Calcineurin axes in pediatric early T-cell precursor ALL. Leukemia, 31, 1007-1011.

Shannan, B., Seifert, M., Leskov, K., Willis, J., Boothman, D., Tilgen, W., \& Reichrath, J. (2006). Challenge and promise: Roles for clusterin in pathogenesis, progression and therapy of cancer. Cell Death Diff, 13, 12-19.

Shi, H., Deng, J. H., Wang, Z., Cao, K. Y., Zhou, L., \& Wan, H. (2013). Knockdown of clusterin inhibits the growth and migration of renal carcinoma cells and leads to differential gene expression. Molecular Medicine Reports, 8, 35-40.

Shim, Y. J., Kang, B. H., Choi, B. K., Park, I. S., \& Min, B. H. (2012). Clusterin induces the secretion of TNF- $\alpha$ and the chemotactic migration of macrophages. Biochemical and Biophysical Research Communications, 422, 200-205.

Shim, Y. J., Kang, B. H., Jeon, H. S., Park, I. S., Lee, K. U., Lee, I. K., ... Min, B. H. (2011). Clusterin induces matrix metalloproteinase- 9 expression via ERK1/2 and PI3K/Akt/NF-kB pathways in monocytes/macrophages. Journal of Leukocyte Biology, 90, 761-769.

Song, M. S., Salmena, L., \& Pandolfi, P. P. (2012). The functions and regulation of the PTEN tumour suppressor. Nature Reviews Molecular Cell Biology, 13, 283-296.

Taylor, B. S., Schultz, N., Hieronymus, H., Gopalan, A., Xiao, Y., Carver, B. S., ... Gerald, W. L. (2010). Integrative genomic profiling of human prostate cancer. Cancer Cell, 18, 11-22. 
Vazquez, F., Ramaswamy, S., Nakamura, N., \& Sellers, W. R. (2000). Phosphorylation of the PTEN tail regulates protein stability and function. Molecular and Cellular Biology, 20, 5010-5018.

Viard, I., Wehrli, P., Jornot, L., Bullani, R., Vechietti, J. L., French, L. E., ... Tschopp, J. (1999). Clusterin gene expression mediates resistance to apoptotic cell death induced by heat shock and oxidative stress. Journal of Investigative Dermatology, 112, 290-296.

Virtakoivu, R., Pellinen, T., Rantala, J. K., Perälä, M., \& Ivaska, J. (2012). Distinct roles of AKT isoforms in regulating $\beta 1$-integrin activity, migration, and invasion in prostate cancer. Molecular Biology of the Cell, 23, 3357-3369.

Wang, C., Jiang, K., Kang, X., Gao, D., Sun, C., Li, Y., ... Liu, Y. (2012). Tumorderived secretory clusterin induces epithelial-mesenchymal transition and facilitates hepatocellular carcinoma metastasis. International Journal of Biochemistry \& Cell Biology, 44, 2308-2320.

Wang, Q., Cao, W., Su, Q., Liu, Z., \& Zhang, L. (2014). Clusterin silencing inhibits proliferation and reduces invasion in human laryngeal squamous carcinoma cells. World Journal of Surgical Oncology, 12, 124-131.

Wang, X., Luo, L., Dong, D., Yu, Q., \& Zhao, K. (2014). Clusterin plays an important role in clear renal cell cancer metastasis. Urologia Internationalis, 92, 95-103.

Zhao, Z., Robinson, R. G., Barnett, S. F., Defeo-Jones, D., Jones, R. E., Hartman, G. D., ... Lindsley, C. W. (2008). Development of potent, allosteric dual Akt1 and Akt2 inhibitors with improved physical properties and cell activity. Bioorganic \& Medicinal Chemistry Letters, 18, 49-53.

Zhong, B., Sallman, D. A., Gilvary, D. L., Pernazza, D., Sahakian, E., Fritz, D., ... Djeu, J. Y. (2010). Induction of clusterin by AKT-role in cytoprotection against docetaxel in prostate tumor cells. Molecular Cancer Therapeutics, 9, 1831-1841.

Zhou, J., Chen, X., Gilvary, D. L., Tejera, M. M., Eksioglu, E. A., Wei, S., \& Djeu, J. Y. (2015). HMGB1 induction of clusterin creates a chemoresistant niche in human prostate tumor cells. Scientific Reports, 5, 15085.

\section{SUPPORTING INFORMATION}

Additional supporting information may be found online in the Supporting Information section at the end of the article.

How to cite this article: Bertacchini J, Mediani L, Beretti F, et al. Clusterin enhances AKT2-mediated motility of normal and cancer prostate cells through a PTEN and PHLPP1 circuit. J Cell Physiol. 2018;1-12. https://doi.org/10.1002/jcp.27768 